\title{
INIBIÇÃO DOS MOVIMENTOS DOS MEMBROS SUPERIORES NA FORÇA VERTICAL DURANTE A MARCHA HUMANA
}

\author{
Inhibition of the movements of upper limbs on the vertical force during the human gait
}

\author{
Aderson Loureiro ${ }^{1,2}$; Andresa Mara de Castro Santos'; Renato Germano'; Milton Antonio Zaro'; \\ Aluisio Otavio Vargas Ávila ${ }^{1,3}$; Tássia Silveira Furlanetto ${ }^{1}$ \\ ${ }^{1}$ IBTeC - Instituto Brasileiro de Tecnologia em Couro Calçados e Afins, Novo Hamburgo - RS \\ ${ }^{2}$ UNISINOS - Universidade do Vale do Rio do Sinos, São Leopoldo - RS \\ ${ }^{3}$ UDESC - Universidade do Estado de Santa Catarina / CEFID / Laboratório de Biomecânica, Florianópolis - SC
}

\begin{abstract}
Resumo: O objetivo deste estudo foi analisar a influência da inibição dos movimentos dos membros superiores na componente vertical da força de reação do solo (CVFRS) durante a marcha descalça, em diferentes velocidades. Participaram voluntariamente da pesquisa 20 sujeitos do gênero feminino com média de idade de $24,1( \pm 3,1)$ anos, fisicamente ativas, sem histórico de lesões nos membros inferiores nos seis meses precedentes às coletas, nem anormalidades nos pés. As variáveis cinéticas estudadas foram: Primeiro Pico de Força (PPF), Suporte Médio (SM) e Segundo Pico de Força (SPF). A marcha descalça foi realizada em duas condições: com inibição dos movimentos dos membros superiores (MI) e marcha normal (MN). O estudo foi realizado no Laboratório de Biomecânica do IBTeC, Novo Hamburgo - RS. Foram utilizadas duas plataformas de força, freqüência de aquisição de $2000 \mathrm{~Hz}$ e foi desenvolvida uma rotina para a aquisição dos dados no programa Matlab $5.0 \Theta$. A marcha foi realizada em uma passarela com 10 metros de comprimento com velocidade controlada através de um conjunto de fotos-célula, sendo $4 \mathrm{~km} / \mathrm{h} \pm 5 \%$ e $6 \mathrm{~km} / \mathrm{h} \pm 5 \%$. O tratamento estatístico foi composto de estatística descritiva exploratória com teste Shapiro-Wilk e Levene, e teste t de Student $(p \leq 0,05)$. Conclusões: Não foram encontradas diferenças sígnificativas nas variáveis PPF, SM e SPF, comparando as condições de marcha normal e marcha com inibição dos membros superiores, na velocidade de $4 \mathrm{~km} / \mathrm{h} \pm 5 \%$, corroborando com os resultados mostrados na revisão bibliográfica. Entretanto, na velocidade de $6 \mathrm{~km} / \mathrm{h} \pm 5 \%$, foi encontrada diferenças estatisticamente significativas nas variáveis PPF e SM, demonstrando a influência dos membros superiores na marcha. Alem disto, o estudo demonstra que os valores nos picos da componente vertical da força tornam-se maiores com o aumento da velocidade da marcha.
\end{abstract}

Palavras-chave: Biomecânica; Força de reação do solo; Marcha; Membros superiores.

Abstract: The aim of this study was to analyze the influence of inhibition of the movements of upper limbs on the vertical component of ground reaction forces (VCGRF) during the barefoot gait, in different speeds. 20 women were selected with average age of 24,1 ( $\pm 3,1)$ years old, physically active, without report of lesions in the inferior limbs into the six months prior to collections, neither abnormalities in the feet. The kinetic variables were first peak of force (FPF), second peak of force (SPF) and rate acceptance weight (RAT) in the conditions of normal gait (NG) and gait with inhibition of the superior limbs (IG). The study was conduced on the Laboratory of Research in Biomechanics of the IBTeC (Brazilian Institute of the Paved Leather and Devices) Novo Hamburgo-RS. The kinetic variables were collected in two force plates with a sampling rate of $2000 \mathrm{~Hz}$, and the data were acquired and processed with MATLAB® 5.0. The gait was carried out in a footbridge of 10 meters of length and for speed controlling, photocells and chronometers were used (speed of $4 \mathrm{~km} / \mathrm{h} \pm 5 \%$ and $6 \mathrm{~km} / \mathrm{h} \pm 5 \%$ ). The statistical treatment was composed of exploratory descriptive statistics, the normality and homogeneity of the data was checked through the test of Shapiro-Wilk and Levene, respectively. When comparing the situations of normal gait and gait with inhibition of the upper limbs it was used the student $t$ Test, considering $p \leq 0,05$. Conclusions: There were no significant differences among the mean values of FPF, MP and SPF in the speed of $4 \mathrm{~km} / \mathrm{h} \pm 5 \%$, neither in the value of SPF in the speed of $6 \mathrm{~km} / \mathrm{h}$ $\pm 5 \%$ comparing NG and IG. However, the study indicated that by inhibiting the movement of the upper limbs a significant influence appeared on the FPF and RAT at the speed of $6 \mathrm{~km} / \mathrm{h} \pm 5 \%$. At the same time, the work demonstrated that the values of specific vertical forces became greater with an increase of speed.

Keywords: Biomechanics. Ground reaction force. Gait. Upper Limbs

Aceito em 30/08/2010 - Revista de Educação Física 2011 Abr; 151:11-16. Rio de Janeiro - Brasil

\section{INTRODUÇÃO}

A marcha humana é uma característica funcional que acompanha o ser humano desde tenrra idade até o final da vida, como via de regra, passa por estágios distintos conforme a idade avança, e contém uma sequência de eventos comuns nos seres humanos. Entretanto, esta seqüência modifica-se de acordo com as características de cada indivíduo, pois além da idade, sua natureza morfológica, gênero e presença de patologias dentre outros fatores, alteram sua dinâmica, sendo assim, existem numerosos estudos visando identificar parâmetros de medidas sobre a mar- 
cha humana(1).

No campo do controle motor, pesquisas recentes têm investigado os reflexos intersegmentares durante a locomoção, que fornecem informações sobre as conexões neurais entre os centros rítmicos controlando os movimentos dos braços e pernas $^{(2)}$. Em outro estudo(3), os autores concluem que a marcha circular é um complexo jogo de mudanças nos impulsos de reação no solo, cinética e cinemática articular, que alteram a orientação do tronco e por conseqüência o centro de massa.

A locomoção, por exemplo, correr, caminhar ou nadar, requer uma série de movimentos coordenados entre os membros ${ }^{(4)}$. Em contrapartida, a locomoção bípede, ou marcha, é uma tarefa funcional que exige interações complexas e coordenação entre muitas das principais articulações do corpo, particularmente da extremidade inferior, sendo esta locomoção uma atividade cíclica que consiste de duas fases para cada membro, apoio e oscilação ${ }^{(5)}$. Os autores deste último estudo ${ }^{(5)}$ ressaltam que os membros superiores e a cabeça não têm recebido tanta atenção na literatura quanto o tronco e os membros inferiores. Dentro desta perspectiva pouco se sabe da influência dos movimentos dos membros superiores na marcha normal e sua relação com as componentes cinéticas da Força de Reação do Solo (FRS).

Mesmo sendo uma atividade básica do movimento humano não deixa de ser bastante comple$x a$, e ainda que as pesquisas tenham avançado, não obstante, há muito que se desvendar e entender sobre os processos e mecanismos da marcha.

O conhecimento sobre o comportamento das variáveis cinéticas frente à variação da velocidade na marcha descalça com um padrão normal dos membros superiores e a inibição dos movimentos dos membros superiores, nos permite compreender as respostas sobre os padrões biomecânicos. Os parâmetros cinéticos da marcha, embora largamente estudados e pesquisados atualmente por muitos pesquisadores $(6,7,8,9,10,11,12)$, são pouco explorados sobre a interação dos membros superiores, e sua influência sobre estes parâmetros cinéticos e sobre a FRS.

Esta afirmação foi comprovada pela revisão bibliográfica realizada em quatro bases de da- dos durante os meses precedentes às coletas, para identificar artigos nos quais tratassem das relações envolvendo a marcha com inibição dos membros superiores com as FRS.

A busca resultou em um total inicial de 54 referencias, das quais três artigos foram selecionados. Destacamos que nenhum artigo aborda diretamente o objeto deste estudo. Portanto, consideramos que este estudo poderá preencher uma lacuna de conhecimento ainda inexistente.

A importância deste estudo está na abordagem de um assunto que propõe produzir conhecimentos ainda não explorados, para a indústria calçadista, para fisioterapia, terapia ocupacional, medicina e para educação física, e no entendimento da influência dos movimentos dos membros superiores na componente vertical da FRS, bem como o significado da velocidade da marcha em mulheres adultas e como isto ocorre. Portanto, o objetivo do estudo foi analisar a influência da inibição dos movimentos dos membros superiores na componente vertical da força de reação do solo (CVFRS) durante a marcha descalça, em diferentes velocidades.

\section{METODOLOGIA}

Os dados foram coletados no Laboratório de Biomecânica do Instituto Brasileiro de Tecnologia do Couro Calçados e Artefatos (IBTeC), em Novo Hamburgo - RS. Participaram deste estudo uma população de mulheres com idade entre 20 e 30 anos completos, escolhidas de forma não-probabilística intencional. Assim, foram selecionadas 20 sujeitos do gênero feminino, fisicamente ativas, sem histórico de lesões nos membros inferiores nos seis meses anteriores às coletas e sem anormalidades nos pés. Esta pesquisa passou por avaliação e obteve aprovação do Comitê de Ética e Pesquisa da UDESC-SC e todos individuos da amostra assinaram o termo de consentimento livre e esclarecido (TCLE).

Foi utilizado o sistema de dinamometria composto por duas plataformas de força (com sensores do tipo "strain gages") inseridas no solo, em série, com dimensões $(40 \times 50,8) \mathrm{cm}$, freqüência natural em torno de $300 \mathrm{~Hz}$, incerteza de medida 
da ordem de $\pm 20 \mathrm{gf}$, distanciadas uma da outra em $1,6 \mathrm{~cm}$. A taxa de amostragem utilizada foi $2000 \mathrm{~Hz}$ e a aquisição e filtragem dos dados foi realizada por meio de uma rotina desenvolvida no programa Matlab $\otimes 5.0$. A rotina desenvolvida realizou a normalização dos dados com base no peso corporal de cada modelo. Além disso, no tratamento de todos os dados utilizou-se um filtro Butterworth de ordem 5 , com freqüência de corte $100 \mathrm{~Hz}$.

Foram realizadas medidas antropométricas das voluntárias através de procedimentos recomendados pelo Anthropometric Standardization Reference Manual|(13). A coleta ocorreu com a marcha descalça em linha reta imaginária passando sobre as plataformas, colocando um pé em cada plataforma mantendo a velocidade determinada.

As voluntárias passaram por um período de adaptação, no qual deveriam caminhar sobre a passarela, objetivando o contato com a primeira plataforma de força com seu pé dominante, e com a finalidade de familiarização com o instrumento de medida. Em seguida, antes do início de cada coleta era realizada uma coleta de 10 segundos, na posição semi-estática, sobre cada plataforma, a fim de realizar a normalização dos dados em função da massa corporal de cada voluntária. As plataformas foram devidamente taradas, antes das coletas de dados.

Assim, as voluntárias realizaram tantas passagens quantas necessárias, até que fossem coletados 5 ciclos completos de marcha com a velocidade estipulada e 0 acerto dos pés sobre a plataforma. Esse procedimento foi utilizado para as duas condições de marcha descalça: 1- Marcha com inibição dos movimentos dos membros superiores (MI); 2- Marcha normal (MN), avaliando as variáveis Primeiro Pico de Força (PPF), Suporte Médio (SM) e SPF (Segundo Pico de Força).

Para as duas condições foram utilizadas duas faixas de velocidades: $4 \mathrm{~km} / \mathrm{h} \pm 5 \%$ e $6 \mathrm{~km} / \mathrm{h} \pm 5 \%$. A escolha das condições e das velocidades para cada voluntária ocorreu de forma randômica. $\mathrm{Na}$ marcha com inibição dos movimentos dos membros superiores (MI) foi utilizado "camisa para inibição dos movimentos" produzida especialmente para o estudo (FIGURA 1).

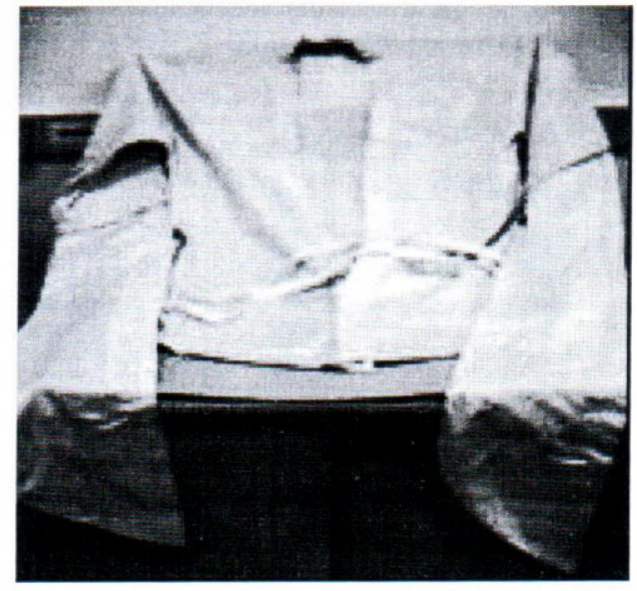

FIGURA 1 - Camisa para inibição dos movimentos (dados do autor).

O sistema utilizado para controle de velocidade foi composto por dois pares de fotos-célula distanciadas $3 \mathrm{~m}$, ligadas a um cronômetro (digital da marca CASIO modelo HS-3 com sensibilidade de 0,001 s), calibrado em laboratório credenciado junto ao INMETRO. Estas fotos-célula ficaram dispostas de tal forma que, com a passagem da modelo através do primeiro par, o cronômetro era ativado, e o mesmo era desativado após a passagem do sujeito através do segundo par. Assim, a velocidade média da pessoa era calculada diretamente na rotina de aquisição de dados, pois se conhecia a distância entre as fotocélulas e o tempo marcado pelo cronômetro.

O tratamento estatístico foi composto de estatística descritiva exploratória, testes de normalidade e homogeneidade (Shapiro-Wilk e Levene). Quanto a comparação entre as situações de marcha normal (MN) e marcha com inibição dos membros (MI) superiores foi utilizado o test $t$ de Student considerando $p \leq 0,05$.

\section{APRESENTAÇÃO E DISCUSSÃO DOS RESULTADOS}

Os valores médios e desvio padrão da Componente Vertical da Força de Reação no Solo (CVFRS), para a velocidade $4 \mathrm{~km} / \mathrm{h} \pm 5 \%$ nas condições MI (marcha com inibição dos movimentos dos membros superiores) e MN (marcha normal), estão apresentados na FIGURA 2. 


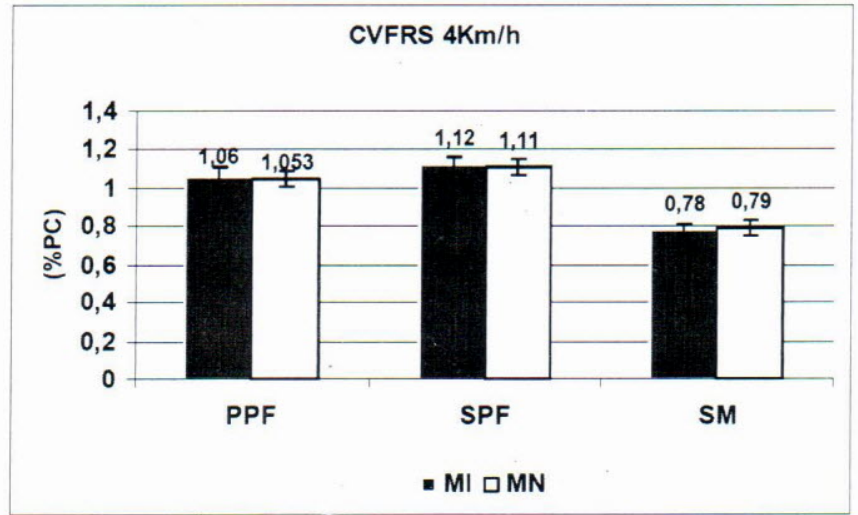

FIGURA 2 - Variáveis cinéticas PPF, SPF e SM nas condições de $\mathrm{Ml}$ e $\mathrm{MN}$ na velocidade de 4 $\mathrm{km} / \mathrm{h} \pm 5 \%$.

O PPF na velocidade de $4 \mathrm{~km} / \mathrm{h} \pm 5 \%$, para a condição $M N$ foi de $(1,05 \pm 0,04 \%)$ do Peso Corporal (PC); já para a condição $\mathrm{Ml}$ o valor médio foi de $(1,06 \pm 0,05 \%)$ do PC. O valor médio de SM foi similar para a condição $M N(0,79 \pm 0,04 \%)$ do $P C$ em relação á condição $\mathrm{MI}(0,78 \pm 0,03 \%)$ do $\mathrm{PC}$. O SPF apresentou discretamente um maior valor médio para a condição $\mathrm{MI}(1,12 \pm 0,04 \%)$ do PC em relação à condição $\mathrm{MN}(1,11 \pm 0,04 \%)$ do $\mathrm{PC}$.

$\mathrm{Na}$ velocidade $6 \mathrm{Km} / \mathrm{h} \pm 5 \%$, os valores médios e desvio padrão da CVFRS, nas condições $\mathrm{MI}$ e MN estão apresentados na FIGURA 3

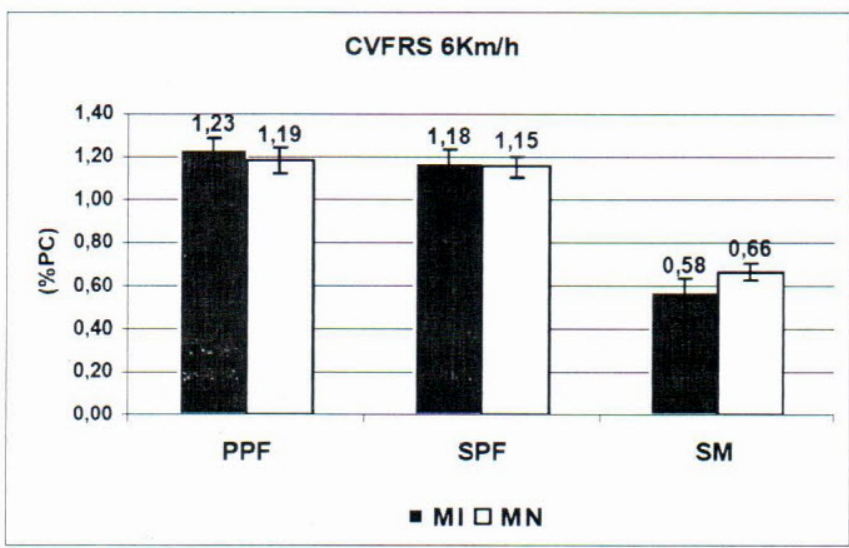

FIGURA 3 - Variáveis cinéticas PPF, SPF e SM nas condições de $\mathrm{MN}$ e $\mathrm{MI}$ na velocidade de 6 $\mathrm{km} / \mathrm{h} \pm 5 \%$.

Os valores médios do PPF à velocidade de $6 \mathrm{~km} / \mathrm{h} \pm 5 \%$ foram superiores aos apresentados na velocidade $4 \mathrm{~km} / \mathrm{h} \pm 5 \%$, sendo para a condi- ção $\mathrm{MN}$ o valor médio foi de $1,19 \pm 0,06 \%$ do $\mathrm{PC}$ e para a condição MI o valor médio foi de 1,23 $\pm 0,06 \%$ do PC. Para o SM, assim como na velocidade a $4 \mathrm{Km} / \mathrm{h} \pm 5 \%$, a condição $\mathrm{MN}$ apresentou maior valor médio $(0,66 \pm 0,04 \%)$ do $\mathrm{PC}$ em relação á condição $\mathrm{MI}(0,58 \pm 0,06 \%)$ do $\mathrm{PC}$. Os valores do SPF foram superiores à velocidade de $4 \mathrm{~km} / \mathrm{h} \pm 5 \%$, sendo que a condição $\mathrm{Ml}$ apresentou maior valor médio $(1,18 \pm 0,06 \%)$ do $\mathrm{PC}$ em relação à condição $M N(1,15 \pm 0,05 \%)$ do $P C$.

Comparando os valores médios entre as variáveis da CVFRS, os achados demonstram que, na velocidade de $4 \mathrm{~km} / \mathrm{h} \pm 5 \%$, não houveram diferenças estatisticamente significativas $(p \leq 0,05)$ entre as condições $\mathrm{MN}$ e $\mathrm{Ml}$ para as variáveis PPF, SPF e SM, corroborando com Ford (2006) ${ }^{(14)}$, o qual afirma que a marcha em baixas velocidades, em torno de $0,8 \mathrm{~m} / \mathrm{s}$ ou aproximadamente $3 \mathrm{~km} / \mathrm{h}$, não influencia nas variáveis analisadas, por existir mínimo balanço dos braços.

O mesmo comportamento ocorreu na comparação dos valores médios da variável SPF da CVFRS, na velocidade de $6 \mathrm{~km} / \mathrm{h} \pm 5 \%$, não foi encontrada diferença estatisticamente significativa entre as condições MN e MI. Entretanto, na comparação do grupo que se locomoveu com inibição dos membros superiores utilizando a camisa de força para tal propósito, e o grupo que se locomoveu com o balanceio normal dos membros superiores, foi mostrado diferenças estatisticamente significativas $(p \leq 0,05)$, para as variáveis PPF e SM.

Estes dados apontam na mesma direção do estudo de Schelee, Andrade e Ávila (2004) $)^{(15)}$, onde reportam o comportamento das vaiáveis PPF, SPF e SM coletados em esteira instrumentalizada para caminhada entre as velocidades de 3 e $7 \mathrm{~km} / \mathrm{h}$, com aumentos de $0,5 \mathrm{~km} / \mathrm{h}$. Os autores concluíram haver diferenças significativas nos valores das variáveis na transição entre velocidades mais elevadas, a partir de $5 \mathrm{~km} / \mathrm{h}^{(15)}$, achados estes que confirmam os achados neste estudo para as diferenças encontradas na velocidade de $6 \mathrm{~km} / \mathrm{h}$ nas variáveis PPF e SM comparando a MI com a $\mathrm{MN}$.

Os dados obtidos deste estudo também estão de acordo com o estudo de Ford $(2007)^{(14)}$, onde 
é salientado que a marcha humana envolve padrões de coordenação específicas entre os segmentos superiores e inferiores que estes são dependentes da velocidade da caminhada.

Analisando os resultados das comparações dos dados apresentados entre o andar na condição de $\mathrm{Ml}$ e o andar na condição de $\mathrm{MN}$, nota-se que o SM apresenta uma deflexão na curva significativamente maior para condição de Ml que na condição de $\mathrm{MN}$, bem como o PPF na condição de $\mathrm{Ml}$ é significativamente maior que na condição de $\mathrm{MN}$, entre outros motivos destacamos que quanto maior a velocidade maior é o balanço dos membros superiores e por conseqüência, maior a influência dos mesmos sobre as variáveis cinéticas estudadas. Sendo assim, estes achados na velocidade de $6 \mathrm{~km} / \mathrm{h} \pm 10 \%$, contrariam alguns estudos $^{(5,16)}$ que afirmaram que os membros superiores não apresentam influência na locomoção humana.

Sobre as respostas das amplitudes das forças verticais na marcha, os resultados neste estudo estão de acordo com os resultado anterior ${ }^{(10)}$, no qual é destacado que estas amplitudes aumentam com a velocidade de 1,0 até 1,5 do peso corporal.

\section{CONCLUSÃO}

Com o presente estudo, que analisou a influência da inibição dos movimentos dos membros superiores durante a marcha em diferentes velocidades, observou-se que a velocidade da caminhada tem influência sobre as variáveis da CVFRS. Assim, houve um aumento nos valores do PPF e SPF, bem como a diminuição do SM, quando aumentado a velocidade da marcha.

Alem disso, os resultados mostram não existir influência dos movimentos dos membros superiores para as variáveis PPF, SPF e SM durante a marcha na velocidade de $4 \mathrm{~km} / \mathrm{h} \pm 10 \%$, corroborando com os poucos estudos na área encontrados. O mesmo ocorreu para o SPF na velocidade de $6 \mathrm{~km} / \mathrm{h} \pm 10 \%$ comparando as condições da MN com a MI. Entretanto, o estudo demostrou que a inibição dos movimentos dos membros superiores influencia significativamente o PPF e o SM na velocidade de $6 \mathrm{~km} / \mathrm{h} \pm 10 \%$.

\section{REFERÊNCIA BIBLIOGRÁFICAS}

1. Taranto J, Taranto MJ, Bryant A, Singer KP. Angle of gait: a comparative reliability study using footprints and the EMED-SF. The Foot 2004; 15: 7-13.

2. Zehr PE, Duysens J. Regulation of arm and leg movement during human locomotion. The Neuroscientist $2004 ; 10$.

3. Orendurff MS, Segal AD, Berge JS, Flick KC, Spanier $D$, Klute GK. The kinematics and kinetics of turning: limb asymmetries associated with walking a circular path. Gait \& Posture 2006; 23: 106-111.

4. Balter JE, Zehr EP. Neural Coupling Between the Arms and Legs During Rhythmic Locomotor-Like Cycling Movement. J Neurophysiol 2007; 97:1809-1818.

5. Nordin M, Frankel VH. Biomecânica básica do sistema esquelético. Rio de Janeiro: Editora Koogan; 2003.

6. Masani K, Kouzaki M, Fukunaga T. Variability of ground reaction forces during treadmill walking. J Appl Physiol 2002; 92:1885-1890.

7. Lelas LJ, Merriman GJ, Riley PO, Kerrigan DC. Predicting peak kinematic and kinetic parameters from gait speed. Gait \& Posture 2003; 17: 106-112.

8. Nurse MA, Hulliger M, Wakeling JM, Nigg BM, Stefanyshyn DJ. Changing the texture of footwear can alter gait patterns. Journal of Electromyography and kinesiology 2005; 15 Issue 5: 496-506.

9. Pain MTG, Challis JH. The influence of soft tissue movement on ground reaction force, joint torques and joint reaction forces in drop landings. Journal of Biomechanics 2006; 39: 119-224.

10. Nilsson J, Thorstensson A. Ground reaction forces at different speeds of human walking and running. Acta Physiologica Scandinavica 2008; 136 Issue 2: 217 227.

11. De Witt JK, Hagan RD, Cromwell RL. The effect of increasing inertial upon vertical ground reaction forces and temporal kinematics during locomotion. The Journal of Experimental Biology 2008; 211: 1087-1092. 
12. Herr $\mathrm{H}$, Popovic M. Angular momentum in human walking. The Journal of Experimental Biology 2007; 211: 467- 481 .

13. Heyward VH, Stolarczyk LM. Avaliação da composição corporal aplicada. São Paulo: Editora Manole, 2000.

14. Ford MP, Wagenaar RC, Newell KM. Arm Constraint and walking in healthy adults. Gait \& Posture 2007; 26 : 135-141.

15. Schelee G, Andrade MC, Avila AOV. Normalização da componente vertical da força de reação no solo para indivíduos não-atletas. Tecnicouro CTCCA 2004; 25:100-103.
16. Perry J. Análise de Marcha. Vol 1. São Paulo: Editora Manole, 2005.

Endereço para correspondência:

Áderson Loureiro

Endereço: Av. Assis Brasil, 2975 - Porto Alegre - RS. CEP: $91010-007$

Telefones: (51) 3361-4140 e 9365-7969

Endereços eletrônicos:

aderson@praxis.esp.br

andresamcs@gmail.com

rg_personaltrainer@yahoo.com.br zaro@ibtec.org.br

biomecanica@ibtec.org.br

tassiasf@gmail.com 